\title{
Magnetic Monopole Cannot Be Incorporated into the Dual Framework of Electromagnetic Theory
}

\author{
Qiankai Yao1,2 \\ ${ }^{1}$ School of Physics and Engineering, Zhengzhou University, Zhengzhou, China \\ ${ }^{2}$ College of Science, Henan University of Technology, Zhengzhou, China \\ Email: yaoqk@zzu.edu.cn
}

Received 2 September 2015; accepted 18 September 2015; published 23 September 2015

Copyright (C) 2015 by author and OALib.

This work is licensed under the Creative Commons Attribution International License (CC BY). http://creativecommons.org/licenses/by/4.0/

(c) (i) Open Access

\begin{abstract}
Through reexamining the intention of duality in classical physics, we will develop the electromagnetic field equations (EFEs) into a new dualized form. In the process, we will see 1) the dual symmetry does not represent the counterpart of electricity and magnetism, but a technique of expression, that is, any dualized equation will be equivalent to its originality. This symmetry can naturally lead to a conserved quantity (called the dual spin), and meanwhile is responsible for charge quantization; 2) magnetic monopole is not compatible with the classical physical laws. Such an opinion can be summarized as no-reality theorem, namely, any Dirac typical dual object will have no reality, such as, no realities of magnetic monopole and permanent electrics, so that the final conclusion is magnetic monopole which does not exist.
\end{abstract}

\section{Keywords}

Magnetic Monopole, 5-D Field Equations, Dual Symmetry, Dual Spin

Subject Area: Theoretical Physics

\section{Introduction}

Regarding the originality of magnetic charge, we could go back to the 19th century. At that time Helmholtz used the concept to calculate the force exerted on a "magnetic particle" by electric current [1]. Meanwhile, based on the fact that the magnetic flux across a closed surface was always zero, Maxwell pointed out that no evidence indicated the possible existence of magnetic charge [2]. Nevertheless, a set of symmetrised MEs was first obtained by Heaviside in 1893 [3], but Heaviside himself didn't trust magnetic charge. Thus, he called it "fictitious", and even thought that its sole worth was providing a convenient way to describe electromagnetic pheno- 
mena. In 1931, Dirac presented an idea of magnetic monopole to symmetrise Maxwell equations (MEs) as

$$
\begin{cases}\nabla \cdot \boldsymbol{E}=\frac{\rho_{\mathrm{e}}}{\varepsilon_{0}}, & \nabla \times \boldsymbol{E}+\frac{\partial \boldsymbol{B}}{\partial t}=-\mu_{0} \boldsymbol{j}_{\mathrm{m}} \\ \nabla \cdot \boldsymbol{B}=\mu_{0} \rho_{\mathrm{m}}, & \nabla \times \boldsymbol{B}-\frac{1}{c^{2}} \frac{\partial \boldsymbol{E}}{\partial t}=\mu_{0} \boldsymbol{j}_{\mathrm{e}}\end{cases}
$$

$\varepsilon_{0}$ and $\mu_{0}$ denote the dielectric permittivity and magnetic permeability. At the same time, Dirac also asserted that magnetic monopole would be responsible for the separate property of charge [4]. Importantly, Dirac's idea could guide people to pursue the natural symmetry. This kind of symmetry is not only in mathematical form but also in physical connotation.

Essentially, what Dirac formulation led to was a Coulomb-like magnetic field with a flux going along a kind of unique string as a channel, but no actual magnetic monopole (at least no experiment could confirm its existence) [5] [6]. After all, breaking two poles (North and South) of a magnet always produced two smaller dipoles, rather than monopoles. Therefore, in Dirac theory, MEs have not really been symmetrised [6]-[8]. This is socalled magnetic monopole question.

Here, by the re-explanation of duality we will highlight that the duality doesn't imply the existence of magnetic monopole, but a more extensive symmetry: MEs are not our only choice, whose dualized form will have the same effectiveness, and thus express the proper dual symmetry of electromagnetic movement. Finally, we will verify that magnetic monopole is impossible.

\section{A General Form of Field Equations}

In modern notation, MEs in Heaviside-Lorentz units read [4] [9]

$$
\begin{cases}\nabla \cdot \boldsymbol{E}=\rho_{\mathrm{e}}, & \nabla \times \boldsymbol{E}+\frac{1}{c} \frac{\partial \boldsymbol{B}}{\partial t}=0 \\ \nabla \cdot \boldsymbol{B}=0, & \nabla \times \boldsymbol{B}-\frac{1}{c} \frac{\partial \boldsymbol{E}}{\partial t}=\frac{\boldsymbol{j}_{\mathrm{e}}}{c}\end{cases}
$$

Applying this equation system in simple medium gives the following non-covariant form

$$
\left\{\begin{array}{lc}
\nabla \cdot \mathbf{E}=\rho_{\mathrm{e}}, & \nabla \times \mathbf{E}+\frac{1}{v_{c}} \frac{\partial \mathbf{B}}{\partial t}=0 \\
\nabla \cdot \mathbf{B}=0, & \nabla \times \mathbf{B}-\frac{1}{v_{c}} \frac{\partial \mathbf{E}}{\partial t}=\frac{\mathbf{j}_{\mathrm{e}}}{v_{c}}
\end{array}, \begin{cases}\mathbf{E}=\sqrt{\varepsilon_{r}} \boldsymbol{E}, & \mathbf{B}=\frac{\boldsymbol{B}}{\sqrt{\mu_{r}}} \\
\rho_{\mathrm{e}}=\frac{\rho_{\mathrm{e}}}{\sqrt{\varepsilon_{r}}}, & \mathbf{j}_{\mathrm{e}}=\frac{\boldsymbol{j}_{\mathrm{e}}}{\sqrt{\varepsilon_{r}}}\end{cases}\right.
$$

with a speed $v_{c}$ defined by relative permittivity $\varepsilon_{r}$ and permeability $\mu_{r}$ as

$$
v_{c}=\frac{c}{\sqrt{\varepsilon_{r} \mu_{r}}}=\frac{c}{\sqrt{\left(1+\chi_{\mathrm{e}}^{\text {med }}\right)\left(1+\chi_{\mathrm{m}}^{\text {med }}\right)}}
$$

$\chi_{\mathrm{e}}^{\mathrm{med}}$ and $\chi_{\mathrm{m}}^{\mathrm{med}}$ denote the electric and magnetic susceptibilities of medium.

A set of covariant equations we want to introduce to replace Equation (2.2), is massive electromagnetic equations (described in Minkowski coordinate system $(x, y, z, \mathfrak{R} \phi, c t))[10]$

$$
\begin{cases}\text { (a) } \nabla \cdot \boldsymbol{E}+\frac{1}{\mathfrak{R}} \frac{\partial b}{\partial \phi}=\rho_{\mathrm{e}}, & \left(\text { b) } \nabla \times \boldsymbol{E}+\frac{1}{c} \frac{\partial \boldsymbol{B}}{\partial t}=0\right. \\ \text { (c) } \nabla \cdot \boldsymbol{B}=0, & \left(\text { d) } \nabla \times \boldsymbol{B}+\frac{1}{\mathfrak{R}} \frac{\partial \boldsymbol{e}}{\partial \phi}-\frac{1}{c} \frac{\partial \boldsymbol{E}}{\partial t}=\frac{\boldsymbol{j}_{\mathrm{e}}}{c}\right. \\ \text { (e) }-\nabla \cdot \boldsymbol{e}-\frac{1}{c} \frac{\partial b}{\partial t}=\frac{\hat{j}_{\mathrm{e}}}{c}, & \text { (f) } \nabla \times \boldsymbol{e}+\frac{1}{\mathfrak{R}} \frac{\partial \boldsymbol{B}}{\partial \phi}=0 \\ \text { (g) } \nabla b-\frac{1}{\mathfrak{R}} \frac{\partial \boldsymbol{E}}{\partial \phi}+\frac{1}{c} \frac{\partial \boldsymbol{e}}{\partial t}=0 & \end{cases}
$$


but need to redefine the polarized vector field $e$ and scalar field $b$

$$
\frac{1}{\Re} \frac{\partial b}{\partial \phi}=\nabla \cdot \boldsymbol{P}_{\mathrm{e}}=-\rho_{\mathrm{e}}, \quad \frac{1}{\Re} \frac{\partial \boldsymbol{e}}{\partial \phi}=-\frac{1}{c} \nabla \times \boldsymbol{M}_{\mathrm{m}}-\frac{1}{c} \frac{\partial \boldsymbol{P}_{\mathrm{e}}}{\partial t}=-\frac{\dot{\boldsymbol{f}}_{\mathrm{e}}}{c}
$$

with

$$
\begin{cases}\boldsymbol{P}_{\mathrm{e}}=\boldsymbol{P}_{\mathrm{e}}^{\mathrm{vac}}+\boldsymbol{P}_{\mathrm{e}}^{\mathrm{med}}, & \boldsymbol{M}_{\mathrm{m}}=\boldsymbol{M}_{\mathrm{m}}^{\mathrm{vac}}+\boldsymbol{M}_{\mathrm{m}}^{\mathrm{med}} \\ \boldsymbol{\rho}_{\mathrm{e}}=\rho_{\mathrm{e}}^{\mathrm{vac}}+\rho_{\mathrm{e}}^{\mathrm{med}}, & \dot{\boldsymbol{f}}_{\mathrm{e}}=\boldsymbol{j}_{\mathrm{e}}^{\mathrm{vac}}+\boldsymbol{j}_{\mathrm{e}}^{\mathrm{med}}\end{cases}
$$

where, the characteristic length $\Re\left(=1.3 \times 10^{26} \mathrm{~m}\right)$ reflects the effective range of electromagnetic interaction. $\boldsymbol{P}_{\mathrm{e}}, \boldsymbol{M}_{\mathrm{m}}$ the total polarization and magnetization (including the vacuum and medium effects), $\boldsymbol{\rho}_{\mathrm{e}}, \dot{\boldsymbol{f}}_{\mathrm{e}}$ the corresponding bounded charge and current. Then, the two susceptibilities become

$$
\varepsilon_{r}=1+\chi_{\mathrm{e}}^{\mathrm{vac}}+\chi_{\mathrm{e}}^{\mathrm{med}}, \quad \mu_{r}=1+\chi_{\mathrm{m}}^{\mathrm{vac}}+\chi_{\mathrm{m}}^{\mathrm{med}}
$$

Now, if consider the charge fluctuation, we are allowed to write the flow equation

$$
\vec{\nabla} \cdot \vec{f}_{\mathrm{e}}+\frac{\partial \rho_{\mathrm{e}}}{\partial t}=0, \quad \vec{\nabla}=\nabla+\frac{1}{\Re} \frac{\partial}{\partial \phi}, \quad \vec{f}_{\mathrm{e}}=\left(\dot{\boldsymbol{j}}_{\mathrm{e}}, \hat{\mathcal{f}}_{\mathrm{e}}\right)
$$

$\vec{\nabla}$ is the gradient operator of 4-dimensional space $(x, y, z, \Re \phi)$, the total added current $\hat{\mathcal{f}}_{\mathrm{e}}\left(=\hat{j}_{\mathrm{e}}^{\mathrm{vac}}+\hat{j}_{\mathrm{e}}^{\mathrm{med}}\right)$ (flowing along $\phi$-axis) consists of two parts: one $\left(\hat{j}_{\mathrm{e}}^{\text {vac }}\right)$ arises from the charge deviation [10], the other $\left(\hat{j}_{\mathrm{e}}^{\text {med }}\right)$ the possible transform between free and bounded charges. Then, the equation together with Equation (2.4) gives

$$
\vec{\nabla} \cdot \vec{j}_{\mathrm{e}}+\frac{\partial \rho_{\mathrm{e}}}{\partial t}=0, \quad \vec{j}_{\mathrm{e}}=\left(\boldsymbol{j}_{\mathrm{e}}, \hat{j}_{\mathrm{e}}\right), \quad \hat{j}_{\mathrm{e}}=-\hat{\mathcal{f}}_{\mathrm{e}}
$$

To illustrate (2.4) covariant, it is sufficient to show that the contained equations can be written entirely in terms of Lorentz tensors. The covariant path will begin with the introduction of electromagnetic field tensor

$$
F^{m n}=\frac{\partial A^{n}}{\partial x_{m}}-\frac{\partial A^{m}}{\partial x_{n}}=A^{n, m}-A^{m, n}=\left[\begin{array}{ccccc}
0 & B_{z} & -B_{y} & e_{x} & -E_{x} \\
-B_{z} & 0 & B_{x} & e_{y} & -E_{y} \\
B_{y} & -B_{x} & 0 & e_{z} & -E_{z} \\
-e_{x} & -e_{y} & -e_{z} & 0 & -b \\
E_{x} & E_{y} & E_{z} & b & 0
\end{array}\right]
$$

with a 5-dimensional potential $A^{m}=(\vec{A}, \varphi)=(A, \hat{A}, \varphi)$. The components of $F^{m n}$ transform according to the generalized Lorentz rule

$$
F^{\prime k}=\alpha_{m}^{l} \alpha_{n}^{k} F^{m n}
$$

with transformation matrix

$$
\alpha_{n}^{m}=\left[\begin{array}{ccc}
\gamma_{u} & \cdots & -\beta_{u} \gamma_{u} \\
\vdots & 1 & \vdots \\
-\beta_{u} \gamma_{u} & \cdots & \gamma_{u}
\end{array}\right], \quad \gamma_{u}=\frac{1}{\sqrt{1-\beta_{u}^{2}}}=\frac{1}{\sqrt{1-u^{2} / c^{2}}}
$$

which reproduces the field transformation formulae

$$
\left\{\begin{array}{l}
E_{/ /}^{\prime}=E_{/ /}, \quad E_{\perp}^{\prime}=\gamma_{u}\left(E_{\perp}+\boldsymbol{\beta}_{u} \times \boldsymbol{B}\right) \\
B_{/ /}^{\prime}=B_{/ /}, \quad B_{\perp}^{\prime}=\gamma_{u}\left(B_{\perp}-\boldsymbol{\beta}_{u} \times \boldsymbol{E}\right) \\
e_{\perp}^{\prime}=e_{\perp}, \quad e_{/ /}^{\prime}=\gamma_{u}\left(e_{/ /}-\beta_{u} b\right) \\
b^{\prime}=\gamma_{u}\left(b-\beta_{u} e_{/ /}\right)
\end{array}\right.
$$

By $F^{m n}$, the homogeneous and non-homogeneous equations in (2.4) can be expressed as 


$$
F^{m n, l}+F^{n l, m}+F^{l m, n}=0, \quad F_{, n}^{m n}=J_{\mathrm{e}}^{m}, \quad J_{\mathrm{e}}^{m}=\left(\vec{j}_{\mathrm{e}} / c, \rho_{\mathrm{e}}\right)
$$

including the generalized continuity equation $J_{\mathrm{e}, m}^{m}=0$ and Lorenz condition $A_{m}^{m}=0$.

\left. In the case of neglecting vacuum effect and added current (i.e. ${\hat{f_{\mathrm{e}}}}_{=0}\right)$, we also have

$$
\left\{\begin{aligned}
\frac{1}{\Re} \frac{\partial b}{\partial \phi} & =\nabla \cdot \boldsymbol{P}_{\mathrm{e}}^{\mathrm{med}}=\chi_{\mathrm{e}}^{\mathrm{med}} \nabla \cdot \boldsymbol{E}=\frac{\chi_{\mathrm{e}}^{\mathrm{med}}}{\sqrt{1+\chi_{\mathrm{e}}^{\mathrm{med}}}} \nabla \cdot \mathbf{E} \\
\frac{1}{\Re} \frac{\partial \boldsymbol{e}}{\partial \phi} & =-\frac{1}{c} \nabla \times \boldsymbol{M}_{\mathrm{mmed}}-\frac{1}{c} \frac{\partial \boldsymbol{P}_{\mathrm{e}}^{\mathrm{med}}}{\partial t}=-\frac{\chi_{\mathrm{m}}^{\mathrm{med}}}{1+\chi_{\mathrm{m}}^{\mathrm{med}}}(\nabla \times \boldsymbol{B})-\frac{\chi_{\mathrm{e}}^{\mathrm{med}}}{c} \frac{\partial \boldsymbol{E}}{\partial t} \\
& =-\frac{\chi_{\mathrm{m}}^{\mathrm{med}}}{\sqrt{1+\chi_{\mathrm{m}}^{\mathrm{med}}}}(\nabla \times \mathbf{B})-\frac{\chi_{\mathrm{e}}^{\mathrm{med}}}{c \sqrt{1+\chi_{\mathrm{e}}^{\mathrm{med}}}} \frac{\partial \mathbf{E}}{\partial t}
\end{aligned}\right.
$$

Substituting them into Equation (2.4), we find its first four equations (a) - (d) just reduce to Equation (2.2), implying the two are completely equivalent. Meanwhile, Equation (e) becomes the bounded charge conservation $\nabla \cdot \boldsymbol{j}_{\mathrm{e}}^{\text {med }}+\partial \rho_{\mathrm{e}}^{\mathrm{med}} / \partial t=0$, and the remainders (g) and (f) acted by operator $\partial / \partial \phi$ will lead to the second field equations respectively

$$
\left\{\begin{array}{l}
\nabla^{2} \boldsymbol{E}_{s}-\frac{1}{c^{2}} \frac{\partial^{2} \boldsymbol{E}_{s}}{\partial t^{2}}=-\frac{1}{\mathfrak{R}^{2}} \frac{\partial^{2} \boldsymbol{E}}{\partial \phi^{2}}=\nabla \rho_{\mathrm{e}}^{\mathrm{med}}+\frac{1}{c^{2}} \frac{\partial \boldsymbol{j}_{\mathrm{e}}^{\mathrm{med}}}{\partial t} \\
\nabla^{2} \boldsymbol{B}_{s}-\frac{1}{c^{2}} \frac{\partial^{2} \boldsymbol{B}_{s}}{\partial t^{2}}=-\frac{1}{\mathfrak{R}^{2}} \frac{\partial^{2} \boldsymbol{B}}{\partial \phi^{2}}=-\frac{1}{c} \nabla \times \boldsymbol{j}_{\mathrm{e}}^{\mathrm{med}}
\end{array}\right.
$$

Correspondingly, the free part obeys

$$
\left\{\begin{array}{l}
\nabla^{2} \boldsymbol{E}_{f}-\frac{1}{c^{2}} \frac{\partial^{2} \boldsymbol{E}_{f}}{\partial t^{2}}=\nabla \rho_{\mathrm{e}}+\frac{1}{c^{2}} \frac{\partial \boldsymbol{j}_{\mathrm{e}}}{\partial t} \\
\nabla^{2} \boldsymbol{B}_{f}-\frac{1}{c^{2}} \frac{\partial^{2} \boldsymbol{B}_{f}}{\partial t^{2}}=-\frac{1}{c} \nabla \times \boldsymbol{j}_{\mathrm{e}}
\end{array}\right.
$$

Pulsing (2.16) and (2.17) together gives

$$
\left\{\begin{array}{l}
\vec{\nabla}^{2} \boldsymbol{E}-\frac{1}{c^{2}} \frac{\partial^{2} \boldsymbol{E}}{\partial t^{2}}=\nabla \rho_{\mathrm{e}}+\frac{1}{c^{2}} \frac{\partial \boldsymbol{j}_{\mathrm{e}}}{\partial t} \\
\vec{\nabla}^{2} \boldsymbol{B}-\frac{1}{c^{2}} \frac{\partial^{2} \boldsymbol{B}}{\partial t^{2}}=-\frac{1}{c} \nabla \times \boldsymbol{j}_{\mathrm{e}}
\end{array},\left\{\begin{array}{l}
\boldsymbol{E}=\boldsymbol{E}_{f}+\boldsymbol{E}_{s} \\
\boldsymbol{B}=\boldsymbol{B}_{f}+\boldsymbol{B}_{s}
\end{array}\right.\right.
$$

Which have the following free wave solutions $\left(\rho_{\mathrm{e}}=\boldsymbol{j}_{\mathrm{e}}=0\right)$

$$
\left\{\begin{array}{l}
\boldsymbol{E} \sim \boldsymbol{E}_{0} \mathrm{e}^{i k_{m} x^{m}} \\
\boldsymbol{B} \sim \boldsymbol{B}_{0} \mathrm{e}^{i k_{m} x^{m}}
\end{array}, k_{m}=\left(\boldsymbol{k}, \frac{\hat{k}_{e f f}}{\mathfrak{R}},-\frac{\omega}{c}\right)\right.
$$

with an effective angular wave number $\hat{k}_{\text {eff }}$ defined by

$$
\widehat{k}_{e f f}=\sqrt{\widehat{k}^{2}+\mathfrak{R}^{2} k_{\mathrm{C}}^{2}}, \quad \hat{k}=\frac{2 \pi}{\hat{\lambda}}=1, \quad k_{\mathrm{C}}=\frac{m_{0} c}{\hbar}
$$

$\hat{k}$ and $k_{\mathrm{C}}$ denote the angular wave number and Compton wave number of photon in medium, $\hat{\lambda}(=2 \pi)$ the angular wave length [10]. Correspondingly, the frequency dependence reads

$$
\omega^{2}=c^{2} k^{2}+\frac{c^{2} \widehat{k}_{e f f}^{2}}{\Re^{2}}=c^{2} k^{2}+\frac{c^{2} \widehat{k}^{2}}{\Re^{2}}+\frac{m_{0}^{2} c^{4}}{\hbar^{2}}
$$

This dispersion relation can help us to get the group and phase velocities 


$$
\left\{\begin{array}{l}
v_{g}=\frac{\mathrm{d} \omega}{\mathrm{d} k}=c \sqrt{1-\frac{c^{2} \widehat{k}_{e f f}^{2}}{\omega^{2} \mathfrak{R}^{2}}}=c \sqrt{1-\left(\frac{c^{2} \widehat{k}^{2}}{\omega^{2} \mathfrak{R}^{2}}+\frac{c^{2} k_{\mathrm{C}}^{2}}{\omega^{2}}\right)} \\
v_{p}=\frac{\omega}{k}=\frac{c}{\sqrt{1-\frac{c^{2} \widehat{k}_{e f f}^{2}}{\omega^{2} \mathfrak{R}^{2}}}}=\frac{c}{\sqrt{1-\left(\frac{c^{2} \widehat{k}^{2}}{\omega^{2} \mathfrak{R}^{2}}+\frac{c^{2} k_{\mathrm{C}}^{2}}{\omega^{2}}\right)}}
\end{array}\right.
$$

The former represents the project of generalized light speed $c$ (propagating in space $(x, y, z, \mathfrak{R} \phi))$ to the real space, the latter the velocity of the intersection point of wave surface and $z$-axis moving along the axis. They both tend to $c$ together, only as $\omega \rightarrow \infty$.

Especially, neglecting magnetization effect (due to $\mu_{r} \sim 1$ for most mediums) gives

$$
v_{g}=\frac{c}{\sqrt{1+\chi_{\mathrm{e}}^{\mathrm{vac}}+\chi_{\mathrm{e}}^{\mathrm{med}}}}=c \sqrt{1-\left(\frac{c^{2} \widehat{k}^{2}}{\omega^{2} \mathfrak{R}^{2}}+\frac{c^{2} k_{\mathrm{C}}^{2}}{\omega^{2}}\right)}
$$

For vacuum case, the group velocity of photon becomes

$$
v_{g}=\frac{c}{\sqrt{1+\chi_{\mathrm{e}}^{\mathrm{vac}}}}=c \sqrt{1-\frac{c^{2} \widehat{k}^{2}}{\omega^{2} \mathfrak{R}^{2}}}
$$

which can help us to estimate the vacuum susceptibility for the radiation of $\omega \sim 10^{15}$ as

$$
\chi_{\mathrm{e}}^{\mathrm{vac}}=\frac{c^{2}}{\mathfrak{R}^{2} \omega^{2}}=\left(\frac{3.0 \times 10^{8}}{1.3 \times 10^{26} \times 10^{15}}\right)^{2}=5.3 \times 10^{-66}
$$

being a very small value. When traveling through a medium of refractive index $n=1.5$, this frequent photon will obtain an inertia mass(corresponding to the spin mass of photon $m_{\gamma}=\hbar /(\Re c)=2.8 \times 10^{-69} \mathrm{~kg}$ ) [10]

$$
m_{0}=\frac{\hbar k_{C}}{\mathrm{c}}=\frac{\hbar \omega}{c^{2}} \sqrt{\left(1-\frac{1}{n^{2}}\right)}=8.6 \times 10^{-37} \mathrm{~kg}=0.5 \mathrm{eV}
$$

only about one-millionth of electron mass $m_{\mathrm{e}}=0.5 \mathrm{MeV}$.

\section{The Dualized Form of Field Equations}

Notice that, by introducing the displacement current $\partial \boldsymbol{E} / \partial t$ in analogy to the magnetic term $\partial \boldsymbol{B} / \partial t$, Maxwell successfully constructed a set of divergence and curl equations both for $\boldsymbol{E}$ and $\boldsymbol{B}$ : it would look like to be quite symmetric, but not completely [7] [9]. To symmetrise (2.4), we introduce the definition of

$$
\begin{cases}\rho_{\mathrm{e}} \rightarrow \rho_{\mathrm{e}}^{*}=\cos \alpha \rho_{\mathrm{e}}, & \rho_{\mathrm{e}}^{\dagger}=-\sin \alpha \rho_{\mathrm{e}} \\ \vec{j}_{\mathrm{e}} \rightarrow \vec{j}_{\mathrm{e}}^{*}=\cos \alpha \vec{j}_{\mathrm{e}}, & \vec{j}_{\mathrm{e}}^{\dagger}=-\sin \alpha \vec{j}_{\mathrm{e}}\end{cases}
$$

for dual current $\left(\vec{j}_{\mathrm{e}}^{\dagger}, \rho_{\mathrm{e}}^{\dagger}\right), \alpha$ is an angular parameter. By which, Equation (2.4) can be expanded equivalently into a 2-component dualized form (superscript * omitted)

$$
\left\{\begin{array}{lll}
\nabla \cdot \boldsymbol{E}+\frac{1}{\mathfrak{R}} \frac{\partial b}{\partial \phi}=\rho_{\mathrm{e}}, & \nabla \cdot \boldsymbol{B}=0, & \nabla \cdot \boldsymbol{e}+\frac{1}{c} \frac{\partial b}{\partial t}=-\frac{\hat{j}_{\mathrm{e}}}{c}, \\
\nabla \cdot \boldsymbol{E}^{\dagger}+\frac{1}{\mathfrak{R}} \frac{\partial b^{\dagger}}{\partial \phi}=\rho_{\mathrm{e}}^{\dagger}, & \nabla \cdot \boldsymbol{B}^{\dagger}=0, & \nabla \cdot \boldsymbol{e}^{\dagger}-\frac{1}{c} \frac{\partial b^{\dagger}}{\partial t}=\frac{\hat{j}_{\mathrm{e}}^{\dagger}}{c} \\
\nabla \times \boldsymbol{E}+\frac{1}{c} \frac{\partial \boldsymbol{B}}{\partial t}=0, & \nabla \times \boldsymbol{B}+\frac{1}{\mathfrak{R}} \frac{\partial \boldsymbol{e}}{\partial \phi}-\frac{1}{c} \frac{\partial \boldsymbol{E}}{\partial t}=\frac{\boldsymbol{j}_{\mathrm{e}}}{c}, & \nabla \times \boldsymbol{e}+\frac{1}{\mathfrak{R}} \frac{\partial \boldsymbol{B}}{\partial \phi}=0 \\
\nabla \times \boldsymbol{E}^{\dagger}-\frac{1}{c} \frac{\partial \boldsymbol{B}^{\dagger}}{\partial t}=0, & -\nabla \times \boldsymbol{B}^{\dagger}-\frac{1}{\mathfrak{R}} \frac{\partial \boldsymbol{e}^{\dagger}}{\partial \phi}-\frac{1}{c} \frac{\partial \boldsymbol{E}^{\dagger}}{\partial t}=\frac{\boldsymbol{j}_{\mathrm{e}}^{\dagger}}{c}, & \nabla \times \boldsymbol{e}^{\dagger}+\frac{1}{\mathfrak{R} \frac{\partial \boldsymbol{B}^{\dagger}}{\partial \phi}=0} \\
\nabla b-\frac{1}{\mathfrak{R}} \frac{\partial \boldsymbol{E}}{\partial \phi}+\frac{1}{c} \frac{\partial \boldsymbol{e}}{\partial t}=0, & \nabla b^{\dagger}-\frac{1}{\mathfrak{R}} \frac{\partial \boldsymbol{E}^{\dagger}}{\partial \phi}-\frac{1}{c} \frac{\partial \boldsymbol{e}^{\dagger}}{\partial t}=0 &
\end{array}\right.
$$


with the dual fields $\boldsymbol{E}^{\dagger}, \boldsymbol{B}^{\dagger}, \boldsymbol{e}^{\dagger}, b^{\dagger}$ given by

$$
\begin{cases}\boldsymbol{E}^{\dagger}=-\nabla \varphi^{\dagger}-\frac{1}{c} \frac{\partial \boldsymbol{A}^{\dagger}}{\partial t}, & \boldsymbol{B}^{\dagger}=-\nabla \times \boldsymbol{A}^{\dagger} \\ \boldsymbol{e}^{\dagger}=-\nabla \hat{A}^{\dagger}+\frac{1}{\mathfrak{R}} \frac{\partial \boldsymbol{A}^{\dagger}}{\partial \phi}, & b^{\dagger}=-\frac{1}{\mathfrak{R}} \frac{\partial \phi^{\dagger}}{\partial \phi}-\frac{1}{c} \frac{\partial \hat{A}^{\dagger}}{\partial t}\end{cases}
$$

$A^{m \dagger}=\left(\boldsymbol{A}^{\dagger}, \widehat{A}^{\dagger}, \varphi^{\dagger}\right)$ is dual to potential $A^{m}=(\boldsymbol{A}, \hat{A}, \varphi)$. The minus sign in front of dual flux $\boldsymbol{j}_{\mathrm{e}}^{\dagger}$ indicates a left hand rule governing its induced fields, in contrast to the right hand rule associated with flux $\boldsymbol{j}_{\mathrm{e}}$. In the way, we get a new set of dualized equations with perfect symmetry, in which electromagnetic fields $(\boldsymbol{E}, \boldsymbol{B}, \boldsymbol{e}, b)$ have the same status completely with their dual objects $\left(\boldsymbol{E}^{\dagger}, \boldsymbol{B}^{\dagger}, \boldsymbol{e}^{\dagger}, b^{\dagger}\right)$. However, it needs to be pointed that, the here defined two groups of field quantities completely different from the original idea of duality, must be required to satisfy different equations: the former obeys the ones without superscript $\dagger$ in Equation (3.2), the latter follows the remainders, that is to possess the mutual independence.

The dual symmetry of Equation (3.2) can be understood as invariance under dual transformation. Thus, by defining the 2-component arrays [11]

$$
\bar{J}^{m}=\left[\begin{array}{c}
J_{\mathrm{e}}^{m} \\
J_{\mathrm{e}}^{m \dagger}
\end{array}\right], \quad \bar{A}^{m}=\left[\begin{array}{c}
A^{m} \\
A^{m \dagger}
\end{array}\right], \quad \bar{F}^{m n}=\bar{A}^{n, m}-\bar{A}^{m, n}=\left[\begin{array}{c}
F^{m n} \\
F^{m n \dagger}
\end{array}\right]
$$

and a matrix

$$
\overline{\bar{U}}(\theta)=\left[\begin{array}{cc}
\cos \theta & \sin \theta \\
-\sin \theta & \cos \theta
\end{array}\right]
$$

we have the transformation relation

$$
\bar{J}^{\prime m}=\overline{\bar{U}} \bar{J}^{m}, \quad \bar{A}^{\prime m}=\overline{\bar{U}} \bar{A}^{m}, \quad \bar{F}^{\prime m n}=\overline{\bar{U}} \bar{F}^{m n}
$$

It shows that, the essence of dual transformation actually reflects a kind of rotation by angle $\theta$. To illustrate the rotation needs to get help from Mobius strip [9], in which if a vector and its anti-vector are designated to represent two dual quantities $\boldsymbol{Q}, \boldsymbol{Q}^{\dagger}$ (see Figure 1), one can find, with translating the vector pair will naturally achieve the exchange of its two components. This means, the distinction between two dual quantities is merely one of definition. So that, if early days the electron was asserted to be dually charged(tantamount to the dual form of MEs first established, or the left hand rule adopted to govern the field of moving charge), then present people would be engaged in analysis of the electrically charged objects, but the result is the same.

In the presented, the electromagnetic fields and their dual objects are treated in the same way, and there some consequences arise. For example, a moving dual charge $q^{\dagger}$ induces $\boldsymbol{B}^{\dagger}$, analogously to a moving electric charge $q$ inducing $\boldsymbol{B}$ (see Figure 2).

The dualized tensor $\bar{F}^{m n}$ can help us to express Equation (3.2) resultantly as

$$
\bar{F}^{m n, l}+\bar{F}^{n l, m}+\bar{F}^{l m, n}=0, \quad \bar{F}_{, n}^{m n}=\bar{J}^{m}
$$

accompanied with the dualized continuity equation $\bar{J}_{, m}^{m}=0$ and Lorenz condition $\bar{A}_{, m}^{m}=0$. Now, it is easy to verify, the developed equations still have gauge invariance under the transformation of $\bar{A}^{\prime m} \rightarrow \bar{A}^{m}+\bar{\varepsilon}^{, m}$, namely

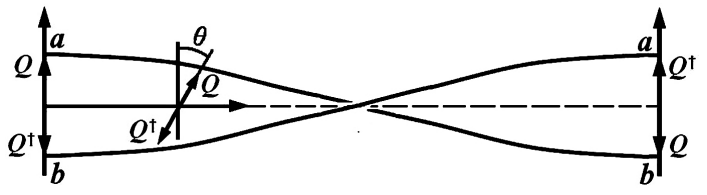

Figure 1. Dual transformation can be illustrated by the translation of dualized vector $\left(\boldsymbol{Q}, \boldsymbol{Q}^{\dagger}\right)$ in the space of Mobius strip (unfolded). 


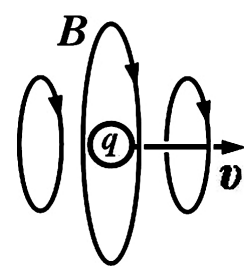

(a)

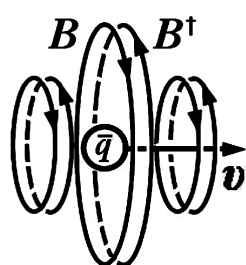

(b)

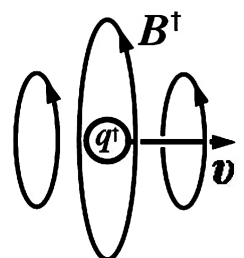

(c)

Figure 2. The fields induced by moving charged particles: (a) A pure electric charge $q, \theta=0$; (b) A dyon (quoted from Schwinger's invention [12]) of carrying two mutual dual charges $\left(q, q^{\dagger}\right), 0<\theta<\pi / 2$; (c) A pure dual charge $q^{\dagger}, \theta=\pi / 2$.

$$
\left\{\begin{array}{l}
\overline{\overrightarrow{\boldsymbol{A}}} \rightarrow \overline{\overrightarrow{\boldsymbol{A}}}+\vec{\nabla} \bar{\varepsilon} \\
\bar{\varphi}^{\prime} \rightarrow \bar{\varphi}-\frac{1}{c} \frac{\partial \bar{\varepsilon}}{\partial t}, \quad \bar{\varepsilon}=\left[\begin{array}{c}
\varepsilon \\
\varepsilon^{\dagger}
\end{array}\right]
\end{array}\right.
$$

$\bar{\varepsilon}$ is an arbitrary dualized scalar function. If gauge invariance held, the Lagrangian reads

$$
L=-\frac{1}{4} \tilde{\bar{F}}_{m n} \bar{F}^{m n}-\tilde{\bar{A}}_{m} \bar{J}^{m}
$$

whose variation with respect to $\vec{A}_{m}$ yields $\bar{F}_{, n}^{m n}=\bar{J}^{m}$. In order to organize effectively EFEs, we write the stress of current $\bar{J}^{m}$ as

$$
f^{m}=\tilde{\bar{F}}^{m n} \bar{J}_{n}=T_{, n}^{m n}
$$

with the electromagnetic stress-energy tensor given by

$$
\left\{\begin{array}{l}
T^{m n}=\eta_{k l} \tilde{\bar{F}}^{m k} \bar{F}^{n l}-\eta^{m n} \Pi \\
\Pi=\frac{1}{4} \tilde{\bar{F}}^{m n} \bar{F}_{m n}=\frac{1}{2}(\tilde{\overline{\boldsymbol{E}}} \cdot \overline{\boldsymbol{E}}-\tilde{\overline{\boldsymbol{B}}} \cdot \overline{\boldsymbol{B}})+\frac{1}{2}(\tilde{\bar{b} b}-\tilde{\overline{\boldsymbol{e}}} \cdot \overline{\boldsymbol{e}})
\end{array}\right.
$$

Applying dual transformation to Equation (3.10), we find

$$
f^{\prime m}=T_{, n}^{\prime m n}=\tilde{\bar{F}}^{\prime m n} \bar{J}_{n}^{\prime}=\overline{\bar{U}} \tilde{\bar{F}} \bar{F}^{m n} \overline{\bar{U}} \bar{J}_{n}=\tilde{\bar{F}}^{m n} \tilde{\bar{U}} \overline{\bar{U}} \bar{J}_{n}=\tilde{\bar{F}}^{m n} \bar{J}_{n}=T_{, n}^{m n}=f^{m}
$$

It shows that, the transformation doesn't change the form of Equation (3.2), and the electromagnetic interaction also keeps the same. In this way, the electrodynamics displays its unique dual invariance. The invariance means, any one would obtain the same results, when he wish to adopt dualized equations to describe the electromagnetic phenomena.

It should be pointed that, due to dual symmetry, the ratio between two dual charges can be arbitrarily chosen, while the physical results will keep the same. So that we say, Equations (2.4) and (3.2) have the equivalent description efficacy; the only difference between them is that, the latter is understanding a charged particle as a dyon of carrying two mutual dual charges, rather than pure electric charge. In other words, regardless of whether an electron is a particle with unit negative charge -e, or a dyon with dualized charge $\bar{q}^{\prime}$ designated by the transformation of

$$
\bar{q}^{\prime}=\overline{\bar{U}}(\theta) \bar{q}=\left[\begin{array}{cc}
\cos \theta & \sin \theta \\
-\sin \theta & \cos \theta
\end{array}\right]\left[\begin{array}{c}
-\mathrm{e} \\
0
\end{array}\right]=\left[\begin{array}{c}
-\cos \theta \mathrm{e} \\
\sin \theta \mathrm{e}
\end{array}\right]
$$

as long as assuming all particles in the universe have the same ratio - $\tan \theta$, the physical results would be unanimous. In particularly, for two "electrons" with charge $\vec{q}^{\prime}$, the "Coulomb" force between them should be

$$
\boldsymbol{f}_{\mathrm{C}}=\frac{1}{4 \pi} \frac{\tilde{\bar{q}} \bar{q}}{r^{3}} \boldsymbol{r}=\frac{1}{4 \pi} \frac{\mathrm{e}^{2}}{r^{3}}\left(\cos ^{2} \theta+\sin ^{2} \theta\right) \boldsymbol{r}=\frac{1}{4 \pi} \frac{\mathrm{e}^{2}}{r^{3}} \boldsymbol{r}
$$


just equal to that between two conventional electrons. This means, Maxwell's theory itself does not be short of symmetry, it can be dualized at any time. After all, we have no absolute reason to say, an electron only carries an unit negative charge $-\mathrm{e}$, it can actually be understood as a dyon of carrying dualized charge $(-\cos \theta \mathrm{e}, \sin \theta \mathrm{e})$, just like in mechanics, a force $\boldsymbol{F}$ is always allowed to be equivalently decomposed into two components, i.e. $\boldsymbol{F}=\boldsymbol{F}_{1}+\boldsymbol{F}_{2}$.

To examine the elements of $T^{m n}$, we find

$$
\left\{\begin{array}{lr}
T^{i j}=\tilde{\bar{E}}^{i} \bar{E}^{j}+\tilde{\bar{B}}^{i} \bar{B}^{j}-\tilde{\bar{e}}^{i} \bar{e}^{j}-\frac{\eta^{i j}}{2}\left(\bar{E}^{2}+\bar{B}^{2}+\bar{b}^{2}-\bar{e}^{2}\right) \\
T^{i 4}=-\frac{\hat{\boldsymbol{S}}}{c}=\tilde{\overline{\boldsymbol{e}}} \times \overline{\boldsymbol{B}}+\tilde{\bar{b}} \overline{\boldsymbol{E}}, & T^{\mu 5}=-\frac{\vec{S}}{c}=(-(\tilde{\overline{\boldsymbol{E}}} \times \overline{\boldsymbol{B}}+\tilde{\bar{b}} \tilde{\boldsymbol{e}}),(\tilde{\bar{E}} \cdot \overline{\boldsymbol{e}})) \\
T^{44}=-\frac{1}{2}\left(\bar{E}^{2}-\bar{B}^{2}-\bar{b}^{2}+\bar{e}^{2}\right), & T^{55}=-w=-\frac{1}{2}\left(\bar{E}^{2}+\bar{B}^{2}+\bar{b}^{2}+\bar{e}^{2}\right)
\end{array}\right.
$$

With this tensor in hand, it is straightforward to confirm that Equation (3.10) contains two conservation laws. The first is a statement of momentum conservation

$$
\vec{f}=\left(\begin{array}{l}
\boldsymbol{f} \\
\hat{f}
\end{array}\right)=\left(\begin{array}{c}
\frac{1}{c}(\tilde{\overline{\boldsymbol{j}}} \times \overline{\boldsymbol{B}}+\tilde{\overline{\bar{j}}} \overline{\boldsymbol{e}})+\tilde{\bar{\rho}} \overline{\boldsymbol{E}} \\
-\frac{1}{c} \tilde{\overline{\boldsymbol{j}}} \cdot \overline{\boldsymbol{e}}+\tilde{\bar{\rho}} \bar{b}
\end{array}\right)=\vec{\nabla} \cdot \overrightarrow{\vec{T}}-\frac{1}{c^{2}} \frac{\partial \vec{S}}{\partial t}
$$

The second gives Poynting's theorem of energy conservation

$$
\vec{f} \cdot \vec{v}=\tilde{\overline{\boldsymbol{j}}} \cdot \overline{\boldsymbol{E}}+\tilde{\overline{\bar{j}}} \overline{\bar{\rho}}=-\vec{\nabla} \cdot \vec{S}-\frac{\partial w}{\partial t}
$$

When no polarized fields $(\boldsymbol{e}, b)$ considered, Equation (3.2) can be merged into a more succinct form

$$
\left\{\begin{array}{l}
\nabla \cdot(\overline{\boldsymbol{E}}+\overline{\boldsymbol{B}})=\bar{\rho} \\
\nabla \times\left(\overline{\boldsymbol{E}}+\overline{\bar{\delta}}_{ \pm} \overline{\boldsymbol{B}}\right)-\frac{1}{c} \frac{\partial\left(\overline{\boldsymbol{E}}-\overline{\bar{\delta}}_{ \pm} \overline{\boldsymbol{B}}\right)}{\partial t}=\frac{\overline{\boldsymbol{j}}}{c}
\end{array}, \overline{\bar{\delta}}_{ \pm}=\left[\begin{array}{cc}
+1 & 0 \\
0 & -1
\end{array}\right]\right.
$$

followed by a 4-force

$$
\left\{\begin{array}{l}
\boldsymbol{f}=\frac{1}{c} \tilde{\overline{\boldsymbol{j}}} \times \overline{\boldsymbol{B}}+\tilde{\bar{\rho}} \overline{\boldsymbol{E}}=\nabla \cdot \overrightarrow{\mathbf{T}}-\frac{1}{c} \frac{\partial(\tilde{\overline{\boldsymbol{E}}} \times \overline{\boldsymbol{B}})}{\partial t} \\
\boldsymbol{f} \cdot \boldsymbol{v}=\tilde{\overline{\boldsymbol{j}}} \cdot \overline{\boldsymbol{E}}=-\nabla \cdot(c \tilde{\overline{\boldsymbol{E}}} \times \overline{\boldsymbol{B}})-\frac{\partial}{\partial t}\left(\frac{\tilde{\overline{\boldsymbol{E}}} \cdot \overline{\boldsymbol{E}}+\tilde{\overline{\boldsymbol{B}}} \cdot \overline{\boldsymbol{B}}}{2}\right)
\end{array}\right.
$$

In absence of dual charge, we get back Maxwell theory.

\section{Conserved Quantity and Charge Quantization}

A celebrated theorem due to Noether makes a general connection: every invariance (or symmetry) of variable transformation would determine a conserved quantity [13]. What is the conserved quantity of dual transformation? It is the dual spin. So that, we focus our interest on the covariant electromagnetic action, and vary an action with the general form

$$
\begin{aligned}
\delta I^{\prime} & =\int \delta L^{\prime}\left(\bar{A}_{m}^{\prime}(\theta), \bar{A}_{m, n}^{\prime}(\theta)\right) \mathrm{d} t \mathrm{~d}^{3} x=\int\left[\frac{\partial L^{\prime}}{\partial \bar{A}_{m}^{\prime}} \frac{\partial \bar{A}_{m}^{\prime}}{\partial \theta}+\frac{\partial L^{\prime}}{\partial \bar{A}_{m, n}^{\prime}} \frac{\partial \bar{A}_{m, n}^{\prime}}{\partial \theta}\right] \delta \theta \mathrm{d} t \mathrm{~d}^{3} x \\
& =\int \frac{\partial}{\partial x^{n}}\left[\frac{\partial L^{\prime}}{\partial \bar{A}_{m, n}^{\prime}} \frac{\partial \bar{A}_{m}^{\prime}}{\partial \theta}\right] \delta \theta \mathrm{d} t \mathrm{~d}^{3} x=0
\end{aligned}
$$

$\mathrm{d}^{3} x$ is the three-dimensional volume element. It requires 


$$
\left\{\begin{array}{l}
\frac{\partial}{\partial x^{n}}\left[\frac{\partial L^{\prime}}{\partial \bar{A}_{m, n}^{\prime}} \frac{\partial \bar{A}_{m}^{\prime}}{\partial \theta}=\tilde{\bar{F}}^{n m} \stackrel{\overline{\bar{U}}}{\frac{\partial \bar{U}}{\partial \theta}} \bar{A}_{m}=\left[F^{n m}, 0^{n m}\right]\left[\begin{array}{cc}
0 & 1 \\
-1 & 0
\end{array}\right]\left[\begin{array}{c}
A_{m} \\
0_{m}
\end{array}\right]\right]=\Theta_{, n}^{n}=0 \\
\Theta^{n}=\left\{\begin{array}{l}
\boldsymbol{\Theta} / c=\left(\mathbf{0}_{\boldsymbol{A}^{\dagger}} \times \boldsymbol{B}-\boldsymbol{A} \times \mathbf{0}_{\boldsymbol{B}^{\dagger}}\right)+c\left(0_{\hat{A}^{\dagger}} \boldsymbol{e}-\hat{A} \mathbf{0}_{\boldsymbol{e}^{\dagger}}\right)+c\left(0_{\varphi^{\dagger}} \boldsymbol{E}-\boldsymbol{\varphi}_{\boldsymbol{E}^{\dagger}}\right)=\mathbf{0} \\
\widehat{\Theta} / c=\left(\mathbf{0}_{\boldsymbol{A}^{\dagger}} \cdot \boldsymbol{e}-\boldsymbol{A} \cdot \mathbf{0}_{\boldsymbol{e}^{\dagger}}\right)+c\left(0_{\varphi^{\dagger}} b-\phi 0_{b^{\dagger}}\right)=0 \\
\vartheta=\left(\mathbf{0}_{\boldsymbol{A}^{\dagger}} \cdot \boldsymbol{E}-\boldsymbol{A} \cdot \mathbf{0}_{\boldsymbol{E}^{\dagger}}\right)+\left(0_{\hat{A}^{\dagger}} b-\hat{A} 0_{b^{\dagger}}\right)=0
\end{array}\right.
\end{array}\right.
$$

and hence, there be the following differential form

$$
\frac{\mathrm{d} \vartheta}{\mathrm{d} t}=\frac{\partial \vartheta}{\partial t}+\vec{\nabla} \vartheta \cdot \frac{\partial \vec{x}}{\partial t}=\frac{\partial \vartheta}{\partial t}+\vec{\nabla}\left(\vartheta \frac{\partial \vec{x}}{\partial t}\right)=\frac{\partial \vartheta}{\partial t}+\vec{\nabla} \cdot \vec{\Theta}=0
$$

which provides a conserved quantity $\vartheta$, called the dual spin density for its arising from the symmetry of dual rotation. We here emphasize, as a "fictitious" rotation, the dual transformation would not lead to any physical effect, and thus determine a zero dual spin of photon, i.e. $\vartheta=0$. On the other hand, due to the introduction of dual quantities increasing the freedom of electromagnetic motion, there needs to supply a new condition to restrict, this condition is nothing but the conversation form $\Theta_{, m}^{m}=0$, just corresponding to $\bar{J}_{, m}^{m}=0$ and $\bar{A}_{, m}^{m}=0$.

To demonstrate charge quantization, we look upon an electrically charged particle with $q$ as a dyon of carrying

$$
\vec{q}=\overline{\bar{U}} \bar{q}=\left[\begin{array}{cc}
\cos \theta & \sin \theta \\
-\sin \theta & \cos \theta
\end{array}\right]\left[\begin{array}{l}
q \\
0
\end{array}\right]=\left[\begin{array}{c}
\cos \theta q \\
\sin \theta \sigma q^{\dagger}
\end{array}\right]
$$

here, a proportional relation of $q=-\sigma q^{\dagger}$ (with an adjustable parameter $\sigma$ ) is supposed. When the dyon moves along the closed Landau orbit $l$ in dualized magnetic fields $\left(\cos \theta \boldsymbol{B}, \sin \theta \sigma \boldsymbol{B}^{\dagger}\right)$, its phase shift arising from the potential $\overline{\boldsymbol{A}}^{\prime}$ should be

$$
\begin{aligned}
\Delta \phi^{\prime} & =\frac{\tilde{\bar{q}}^{\prime}}{\hbar c} \oint_{l} \overline{\boldsymbol{A}}^{\prime} \cdot \mathrm{d} \boldsymbol{r}=\frac{\tilde{\bar{q}}^{\prime}}{\hbar c} \iint_{S_{l}}\left(\nabla \times \overline{\boldsymbol{A}}^{\prime}\right) \cdot \mathrm{d} \boldsymbol{S}_{l}=\frac{\tilde{\bar{q}}^{\prime}}{\hbar c} \bar{\Phi}_{\bar{B}}^{\prime} \\
& =\frac{1}{\hbar c}\left(\cos ^{2} \theta q \Phi_{B}+\sin ^{2} \theta \sigma^{2} q^{?} \Phi_{B^{\dagger}}\right)=2 \pi(1,2,3, \cdots),
\end{aligned}
$$

$\bar{\Phi}_{\bar{B}}^{\prime}$ is the flow of $\overline{\boldsymbol{B}}^{\prime}$ through any surface $S_{l}$ bounded by $l$. The dual covariance requires

$$
\delta \Delta \phi^{\prime}=\frac{\partial}{\partial \theta}\left(\frac{\tilde{\bar{q}}^{\prime}}{\hbar c} \bar{\Phi}_{\bar{B}}^{\prime}\right) \delta \theta=\frac{1}{\hbar c}\left(-\sin 2 \theta q \Phi_{B}+\sin 2 \theta \sigma^{2} q^{?} \Phi_{B^{\dagger}}\right) \delta \theta=0
$$

Then, based on the independency of two fluxes $\left(\Phi_{B}, \Phi_{B^{\dagger}}\right)$, we deduce

$$
\frac{\Phi_{B}}{q^{\dagger}}=\frac{\sigma^{2} \Phi_{B^{\dagger}}}{q}=\text { const. }
$$

Due to the adjustability of $\sigma$, the constant is always allowed to be unit by redefining $q^{\dagger}$, namely

$$
\Phi_{B}=q^{\dagger}, \quad \sigma^{2} \Phi_{B^{\dagger}}=q
$$

So that, putting the obtained into (4.5) gives the quantization relationship

$$
q q^{\dagger}=n h c, \quad n=1,2,3, \cdots
$$

This derivation implies, not have to get help from Dirac monopole, the dual covariance would naturally lead to the charge quantization. In other words, the root of charge quantization is not at the existence of magnetic monopole, but the dual symmetry of electromagnetic movement. 


\section{Magnetic Monopole Does Not Exist}

Right now, if suppose the dual object of electric charge were Dirac magnetic monopole (i.e. $\rho_{\mathrm{e}}^{\dagger}=\rho_{\mathrm{m}}, \quad \boldsymbol{j}_{\mathrm{e}}^{\dagger}=\boldsymbol{j}_{\mathrm{m}}$ ), and the electromagnetic fields dual each other: $\boldsymbol{E}^{\dagger}=\boldsymbol{B}, \boldsymbol{B}^{\dagger}=-\boldsymbol{E}$, we can reedit Equation (3.2) in the form of

$$
\begin{cases}\nabla \cdot \boldsymbol{E}=\rho_{\mathrm{e}}+\rho_{\mathrm{e}}, & \nabla \times \boldsymbol{E}+\frac{1}{c} \frac{\partial \boldsymbol{B}}{\partial t}=-\frac{\dot{\boldsymbol{j}}_{\mathrm{m}}+\boldsymbol{j}_{\mathrm{m}}}{c} \\ \nabla \cdot \boldsymbol{B}=\rho_{\mathrm{m}}+\rho_{\mathrm{m}}, & \nabla \times \boldsymbol{B}-\frac{1}{c} \frac{\partial \boldsymbol{E}}{\partial t}=\frac{\dot{\boldsymbol{j}}_{\mathrm{e}}+\dot{j}_{\mathrm{e}}}{c} \\ \vec{\nabla} \cdot \overrightarrow{\boldsymbol{f}}_{\mathrm{e}}+\frac{\partial \rho_{\mathrm{e}}}{\partial t}=0, & \vec{\nabla} \cdot \overrightarrow{\dot{f}}_{\mathrm{m}}+\frac{\partial \rho_{\mathrm{m}}}{\partial t}=0\end{cases}
$$

$\rho_{\mathrm{m}}, \overrightarrow{\dot{J}}_{\mathrm{m}}=\left(\dot{\boldsymbol{f}}_{\mathrm{m}}, \hat{\dot{J}}_{\mathrm{m}}\right)$ are the corresponding bounded magnetic charge and current. In the case of no bounded, these equations reduce to the Maxwell-Dirac form (i.e. Equation (1.1))

$$
\begin{cases}\nabla \cdot \boldsymbol{E}=\rho_{\mathrm{e}}, & \nabla \times \boldsymbol{E}+\frac{1}{c} \frac{\partial \boldsymbol{B}}{\partial t}=-\frac{\boldsymbol{j}_{\mathrm{m}}}{c} \\ \nabla \cdot \boldsymbol{B}=\rho_{\mathrm{m}}, & \nabla \times \boldsymbol{B}-\frac{1}{c} \frac{\partial \boldsymbol{E}}{\partial t}=\frac{\boldsymbol{j}_{\mathrm{e}}}{c}\end{cases}
$$

From which, we see that, the electric and magnetic quantities have been put in perfectly symmetrical position, and at present, there would be three kinds of way to produce magnetic field: magnetic charge, moving electric charge and changing electric field. So, the energy-momentum conservation for Maxwell-Dirac system needs to be derived from Equation (5.2), namely

$$
\left\{\begin{array}{l}
\boldsymbol{f}=\frac{1}{c}\left(\boldsymbol{j}_{\mathrm{e}} \times \boldsymbol{B}-\boldsymbol{j}_{\mathrm{m}} \times \boldsymbol{E}\right)+\rho_{\mathrm{e}} \boldsymbol{E}+\rho_{\mathrm{m}} \boldsymbol{B}=\nabla \cdot \overrightarrow{\mathbf{T}}-\frac{1}{c^{2}} \frac{\partial \boldsymbol{S}}{\partial t} \\
\boldsymbol{f} \cdot \boldsymbol{v}=\boldsymbol{j}_{\mathrm{e}} \cdot \boldsymbol{E}+\boldsymbol{j}_{\mathrm{m}} \cdot \boldsymbol{B}=-\nabla \cdot \boldsymbol{S}-\frac{\partial w}{\partial t}
\end{array}\right.
$$

Just as our expectation, the electromagnetic energy-momentum changes into the mechanical form, in turn, the latter gets to balance by the former reducing. Ostensibly, it seems to imply, the law of energy-momentum conservation is still working well, and Maxwell-Dirac theory has no question, at least in mathematical form, not conflict with the classical physics. However, this is not the case; the involvement of magnetic monopole will completely change the trend of whole theory.

To illustrate this point, we suppose the existence of magnetic monopole, and conceive an electromagnetic system. As shown in Figure 3, the system is consisting of a permanent magnet and a magnetic circuit $\left(\rho_{\mathrm{e}}=\boldsymbol{j}_{\mathrm{e}}=0\right)$,

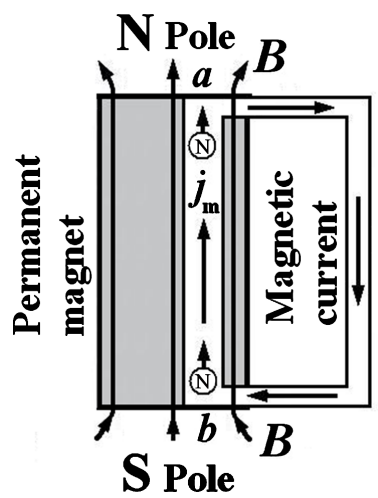

Figure 3. An electromagnetic system is supposed to be composed of magnetic current $\boldsymbol{j}_{\mathrm{m}}$ flowing through the rotational magnetostatic field $\boldsymbol{B}$ caused by a permanent magnet. Then analogy the usual Lorentz force, there should be a corresponding formula for magnetic charge movement, that is a continuous magnetic force $\boldsymbol{f}_{\mathrm{m}}$. The appearance of $\boldsymbol{f}_{\mathrm{m}}$ implies, the electromagnetic energy-momentum seems to be able to transform unlimitedly into the mechanical form. This obviously violates the basic physical law. 
when there be a flux $\boldsymbol{j}_{\mathrm{m}}$ flowing through the circuit, the rotational magnetostatic field $\boldsymbol{B}$ caused by the magnet will exert a stress on it, i.e. $\boldsymbol{f}_{\mathrm{m}}=-\boldsymbol{j}_{\mathrm{m}} \times \boldsymbol{E} / c+\rho_{\mathrm{m}} \boldsymbol{B}$. Then, by Equation (5.3) we can write the following integral over the surface $\Sigma$ that bounds $V$

$$
\left\{\begin{array}{l}
\iiint_{V} \boldsymbol{f}_{\mathrm{m}} \mathrm{d} V=\iiint_{V}\left(-\frac{1}{c} \boldsymbol{j}_{\mathrm{m}} \times \boldsymbol{E}+\rho_{\mathrm{m}} \boldsymbol{B}\right) \mathrm{d} V=\oiint_{\Sigma} \overrightarrow{\mathbf{T}} \cdot \mathrm{d} \boldsymbol{\Sigma}-\frac{1}{c^{2}} \frac{\partial}{\partial t} \iiint_{V} \boldsymbol{S} \mathrm{d} V \\
\iiint_{V} \boldsymbol{f} \cdot \boldsymbol{v} \mathrm{d} V=\iiint_{V}\left(\boldsymbol{j}_{\mathrm{m}} \cdot \boldsymbol{B}\right) \mathrm{d} V=-\oiint_{\Sigma} \boldsymbol{S} \cdot \mathrm{d} \boldsymbol{\Sigma}-\frac{\partial}{\partial t} \iiint_{V} w \mathrm{~d} V
\end{array}\right.
$$

For unbounded space(modeled as a spherical volume tending to infinity), no momentum and energy in or out the closed boundary $\Sigma$, and the conservation equations become

$$
\left\{\begin{array}{l}
\boldsymbol{P}_{\text {mech }}=\int \boldsymbol{F}_{\mathrm{m}} \mathrm{d} t=\boldsymbol{P}_{0}-\frac{1}{c^{2}} \iiint_{V} \boldsymbol{S} \mathrm{d} V=\boldsymbol{P}_{0}-\boldsymbol{P}_{\mathrm{em}} \\
E_{\text {mech }}=\int W_{\mathrm{m}} \mathrm{d} t=E_{0}-\iiint_{V} w \mathrm{~d} V=E_{0}-E_{\mathrm{em}}
\end{array}\right.
$$

with the total magnetic force $\boldsymbol{F}_{\mathrm{m}}$ and power $W_{\mathrm{m}}$ given by

$$
\left\{\begin{array}{l}
\boldsymbol{F}_{\mathrm{m}}=\iiint_{V} \rho_{\mathrm{m}} \boldsymbol{B} \mathrm{d} V=\rho_{\mathrm{m}} V_{a b} \boldsymbol{B} \\
W_{\mathrm{m}}=\iiint_{V}\left(\boldsymbol{j}_{\mathrm{m}} \cdot \boldsymbol{B}\right) \mathrm{d} V=j_{\mathrm{m}} V_{a b} B
\end{array}\right.
$$

$V_{a b}$ denotes the volume of magnetic conductor $\overline{a b}$. It is clear that, the increase of mechanical energy-momentum can always be compensated by the surrounding fields. The conclusion itself has no any question, however this is only an ostensible impression, when $\boldsymbol{F}_{\mathrm{m}}$ and $W_{\mathrm{m}}$ have the steady and finite values, the mechanical momentum and energy of whole system will be inevitable to increase continuously, even tend to infinity

$$
\left\{\begin{array}{l}
\boldsymbol{P}_{\text {mech }}=\left.\boldsymbol{F}_{\mathrm{m}} t\right|_{t \rightarrow \infty} \rightarrow \infty \\
E_{\text {mech }}=\left.W_{\mathrm{m}} t\right|_{t \rightarrow \infty} \rightarrow \infty
\end{array}\right.
$$

These results tell us that, the conceived system could unlimitedly transform its electromagnetic momentum and energy into the mechanical form, even like avalanche to an infinite negative value region(with imaginary fields $\boldsymbol{E} \rightarrow i \boldsymbol{E}, \quad \boldsymbol{B} \rightarrow i \boldsymbol{B})$, namely

$$
\left\{\begin{array}{l}
\boldsymbol{P}_{\mathrm{em}}=\frac{1}{c} \iiint_{V}(i \boldsymbol{E} \times i \boldsymbol{B}) \mathrm{d} V \sim-\left.\boldsymbol{F}_{\mathrm{m}} t\right|_{t \rightarrow \infty} \rightarrow-\infty \\
E_{\mathrm{em}}=\frac{1}{2} \iiint_{V}(i \boldsymbol{E} \cdot i \boldsymbol{E}+i \boldsymbol{B} \cdot i \boldsymbol{B}) \mathrm{d} V \sim-\left.W_{\mathrm{m}} t\right|_{t \rightarrow \infty} \rightarrow-\infty
\end{array}\right.
$$

This is impossible by energy-momentum conservation. Therefore, as long as no physical experiment could detect the above transform, the assumption of that the magnetic monopole exists in universe cannot be true.

Hereby, it can be concluded that, the notion of magnetic monopole is not compatible with the classical physical laws. In other words, the magnetic field is not suitable to be understood as the dual target of electric one, since the magnetism is completely staying in an unequal position to electricity (see Table 1).

From the table we see that, any two supposed dual objects are actually antithetical each other, that is, if either is true (false), the other must be false (true). This situation can be stated as two equivalent statements:

1) There are no physical objects dual each other in Dirac mode, or any Dirac typical dual object has no reality (called no-reality theorem);

2) Any static vortex field is impossible to be an active one, or any field with source is impossible to possess simultaneously a static vortex structure (called no-vortex theorem).

Their equivalence can be proved by showing that if either is false, so is the other. Specifically, if the former were violated, namely magnetic monopole exist, magnetic field will become an active field. At the same time, the rotational magnetostatic field caused by permanent magnet also exists, and thus violates the second statement. In turn, the conclusion remains the same. 
The key point is that, due to possessing a vortex structure, magnetic field lines do not begin or end in any volume of space(reflected completely by $\nabla \cdot \boldsymbol{B}=0$ ). Consequently, if allow the existence of Dirac typical dual objects, one may use them to construct a kind of working system that will produce some unphysical results. For example, whether magnetic monopole or rotational electrostatic field (induced by permanent electrics analogous to permanent magnet) exists, there would be a type of perpetual mobile that can output power unlimitedly, this obviously violates the law of energy conservation, as shown in Figure 4.

In short, Maxwell theory itself does not require or forbid magnetic monopole exists, as well as the impossibility of perpetual mobile of the first kind states that, magnetic monopole has no reality. Although magnetic monopole has no reality, it still does not negate the usefulness of duality as a tool for studying the electromagnetic movement from a viewpoint of symmetry.

\section{Conclusions}

In conclusion, our acquisitions can be summed up as follows:

1) The developed 5-dimensional EFEs can provide us with a unified formalism; this formalism describing the medium effect by independent polarized fields has led to some meaningful results, such as the propagation and dispersion of light in matter;

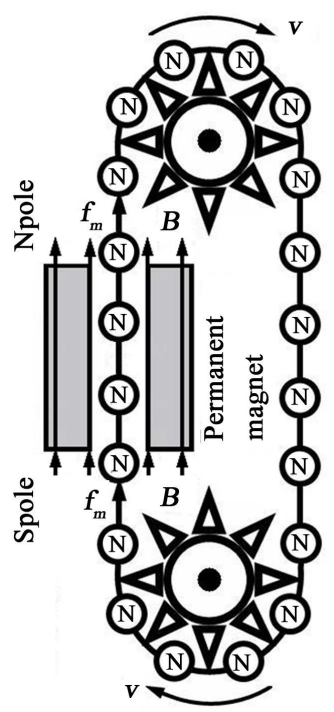

(a)

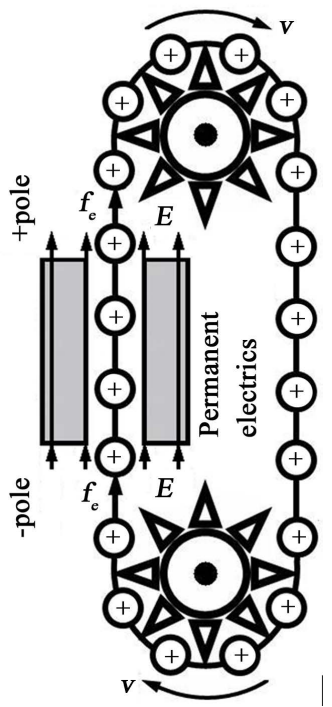

(b)

Figure 4. The existence of magnetic monopole or permanent electrics (the electric analog of permanent magnet) implies the feasibility of perpetual mobile of the first kind. Specifically, when moving through a rotational magnetostatic (a) (electrostatic (b)) field caused by permanent magnet (electrics), the magnetic (electric) charge system will work as a perpetual mobile of outputting the power unlimitedly. This is impossible.

Table 1. Some electromagnetic objects and their statuses $(\sqrt{ }$ and $\times$ denote reality and no reality).

\begin{tabular}{ccccc}
\hline Electricity & Electric charge & $\begin{array}{c}\text { Electric dipole } \\
\text { (placement of poles) }\end{array}$ & $\begin{array}{c}\text { Electric dipole } \\
\text { (loop of current) }\end{array}$ & $\times$ \\
\hline Status & $\sqrt{ }$ & Magnetic dipole & Rotational \\
electrostatic field & Magnetic dipole \\
Magnetism & Magnetic charge & $\times$ & $\sqrt{\text { placement of poles) }}$ & Rotational \\
(loop of current) & Permanent electrics & Spin electric moment \\
\hline Status & $\times$ & $\times$ & Polecular electric current & $\times$ \\
\hline Status & Electric atom & $\sqrt{ }$ & Permanent magnet & Spin magnetic moment \\
\hline Magnetism & Magnetic atom & Molecular magnetic current & $\sqrt{ }$ \\
\hline
\end{tabular}


2) Due to possessing different natures, the electric and magnetic fields are not dual each other, whereas the dual quantities must be independent mutually. Based on the decision, we have developed EFEs into a 2-component dualized form. This form emphasizes that all the dualized equations should have the equivalent description efficacy to its originality. Especially, the dual spin of photon is essentially related to the electromagnetic dual symmetry, which also can provide us with a natural explanation for charge quantization;

3) Magnetic monopole is impossible, since it allows the electromagnetic energy-momentum to be transformed unlimitedly into the mechanical form. Crucially, the motivation of Dirac proposing magnetic monopole is to symmetrise MEs, but here it is no longer an indispensable concept. Eventually, Maxwell theory can be symmetrised at any time; it originally represents a particular form of 2-component dualized EFEs.

\section{References}

[1] Helmholtz, H.V. (1858) Ueber Integrale der hydrodynamischen Gleichungen, welche der Wirbelbewegung entsprechen. Journal für die reine und angewandte Mathematik, 55, 25. (In German)

[2] Maxwell, J.C. (1865) A Dynamical Theory of the Electromagnetic Field. Philosophical Transactions of the Royal Society of London, 155, 459-512. http://dx.doi.org/10.1098/rstl.1865.0008

[3] Heaviside, O. (1893) Electromagnetic Theory. Chelsea Publishing Co., London.

[4] Dirac, P.A.M. (1931) Quantized Singularities in the Electromagnetic Field. Proceedings of the Royal Society A, 133, 60. http://dx.doi.org/10.1098/rspa.1931.0130

[5] Dirac, P.A.M. (1948) The Theory of Magnetic Poles. Physical Review Letters, 74, 817. http://dx.doi.org/10.1103/PhysRev.74.817

[6] Preskill, J. (1984) Magnetic Monopoles. Annual Review of Nuclear and Particle Science, 34, 461-530. http://dx.doi.org/10.1146/annurev.ns.34.120184.002333

[7] Milton, K.A. (2006) Theoretical and Experimental Status of Magnetic Monopoles. Reports on Progress in Physics, 69, 1637. http://dx.doi.org/10.1088/0034-4885/69/6/R02

[8] Rajantie, A. (2012) Introduction to Magnetic Monopoles. Contemporary Physics, 53, 195-211.

[9] Fryberger, D. (1985) Magnetic Monopole. IEEE Transactions on Magnetics, 21, 84-101. http://dx.doi.org/10.1109/TMAG.1985.1063877

[10] Yao, Q.K. (2015) The Unified Theoretical Form of Massive Electrodynamics. Open Access Library Journal, 2 , e1372. http://dx.doi.org/10.4236/oalib.1101732

[11] Gonano, C.A. (2011) Estensione in N-D di prodotto vettore e rotore e loro applicazioni. M.S. Thesis. (In Italy) https://www.politesi.polimi.it/bitstream/10589/34061/1/2011_12_Gonano.pdf, Dec. 2011.

[12] Schwinger, J. (1969) A Magnetic Model of Matter. Science, 165, 757-761. http://dx.doi.org/10.1126/science.165.3895.757

[13] Barut, A.O. (1964) Electrodynamics and Classical Field Theory of Fields and Particles. Macmillan, New York. 